\title{
Alfvén wave interaction with inhomogeneous plasmas: acceleration and energy cascade towards small-scales
}

\author{
V. Génot ${ }^{1, *}$, P. Louarn ${ }^{2}$, and F. Mottez ${ }^{3}$ \\ ${ }^{1}$ Astronomy Unit, Queen Mary, University of London, Mile End Road, London E1 4NS, England, UK \\ ${ }^{2}$ Centre d'Etude Spatiale des Rayonnements, 9 Av. Colonel Roche, 31400 Toulouse, France \\ ${ }^{3}$ Centre d'étude des Environnements Terrestre et Planétaires, 10-12 Av. de l'Europe, 78140 Vélizy, France \\ *Now permanently at CESR, Toulouse, France
}

Received: 4 June 2003 - Revised: 14 January 2004 - Accepted: 28 January 2004 - Published: 14 June 2004

\begin{abstract}
Investigating the process of electron acceleration in auroral regions, we present a study of the temporal evolution of the interaction of Alfvén waves (AW) with a plasma inhomogeneous in a direction transverse to the static magnetic field. This type of inhomogeneity is typical of the density cavities extended along the magnetic field in auroral acceleration regions. We use self-consistent Particle In Cell (PIC) simulations which are able to reproduce the full nonlinear evolution of the electromagnetic waves, as well as the trajectories of ions and electrons in phase space. Physical processes are studied down to the ion Larmor radius and electron skin depth scales. We show that the AW propagation on sharp density gradients leads to the formation of a significant parallel (to the magnetic field) electric field (E-field). It results from an electric charge separation generated on the density gradients by the polarization drift associated with the time varying AW E-field. Its amplitude may reach a few percents of the AW E-field. This parallel component accelerates electrons up to $\mathrm{keV}$ energies over a distance of a few hundred Debye lengths, and induces the formation of electron beams. These beams trigger electrostatic plasma instabilities which evolve toward the formation of nonlinear electrostatic structures (identified as electron holes and double layers). When the electrostatic turbulence is fully developed we show that it reduces the further wave/particle exchange. This sequence of mechanisms is analyzed with the program WHAMP, to identify the instabilities at work and wavelet analysis techniques are used to characterize the regime of energy conversions (from electromagnetic to electrostatic structures, from large to small length scales). This study elucidates a possible scenario to account for the particle acceleration and the wave dissipation in inhomogeneous plasmas. It would consist of successive phases of acceleration along the magnetic field, the development of an electrostatic turbulence, the thermalization and the heating of the plasma.
\end{abstract}

Correspondence to: V. Génot

(vincent.genot@cesr.fr)
Key words. Space plasma physics (charged particle motion and acceleration; numerical studies). Magnetospheric physics (plasma waves and instabilities)

\section{Introduction}

The near-Earth space environment is often considered as a plasma laboratory where it is possible to investigate basic plasma processes thanks to in-situ spacecraft measurements. It is interesting, when it is possible, to extrapolate this knowledge to remote astrophysical environments where in-situ observations are difficult or not yet feasible. In this frame a particularly general and important plasma mechanism which still needs to be elucidated is the particle acceleration in highly magnetized plasma which is characteristic, among others, of the auroral magnetospheric acceleration.

An interesting feature of space plasma is the quite common existence of small-scale structures of the electric field aligned with the ambient magnetic field (on the scale of a few tens of Debye lengths). The formation of these structures, their stability, and their role in the global organization of the plasma and in the energy redistribution between fields and particles, are questions of general interest in space physics. The existence of small-scale E-field structures has long been reported in the literature, in environments as diverse as the solar wind (Mangeney et al., 1999), the terrestrial bow shock (Bale et al., 1998, 2002), the auroral regions (Temerin et al., 1982), and the magnetotail (Omura et al., 1996). They usually appear in situations where particle acceleration is observed. Thus, the observations of auroral features (UV and radio emissions with which they are associated on Earth) on Jupiter (Clarke et al., 2002) suggest that they may also exist in extraterrestrial magnetospheres. Whether these structures are the cause or consequence of the acceleration is not clear. Indeed, particle acceleration in non-collisional plasmas is a long standing issue. Whereas observations show the existence of particle flux with energy reaching a few $\mathrm{keV}$ in 
the terrestrial auroral zone, the natural idea which could explain these flux, consisting of invoking a parallel to the ambient magnetic field E-field, is challenged by the extremely large parallel conductivity of the medium. This conductivity is likely to short-circuit any parallel E-field. Therefore, numerous theories and models have been developed to tackle this challenging question, although searchs for parallel electric fields are not the only path which has been explored. For example, microscopic processes may (i) introduce anomalous resistivity along the auroral magnetic field lines, (Papadopoulos, 1977), (ii) create small-scale non-linear structures (Boström et al., 1988; Mälkki et al., 1993; Mottez et al., 1992), or (iii) directly transfer energy from high frequency waves (Bingham et al., 1984, 1988). On larger scales, global ionosphere/magnetosphere electrostatic potential differences can also develop over the whole auroral field lines, as in the strong double-layer model (Miura and Sato, 1980) or in the kinetic model initially proposed by Alfvén and Fälthammar, (1963); see also Chiu and Schulz (1978).

Although several of those mechanisms may coexist for a given environment, direct observations of parallel electric fields suggest that they likely play an important role in particle acceleration (Andersson et al. 2002a; Ergun 2001). The nature of the electric field configuration which leads to the most efficient acceleration is still controversial. Is it the large-scale (in the $1000 \mathrm{~km}$ range) static fields corresponding to U-shaped potential structures, as revealed by Dynamic Explorer and Viking spacecraft observations, for instance? Those of a wavy nature as Freja spacecraft measurements tend to show? Or the strong double layers measured by Fast spacecraft? However, a general agreement seems now to emerge in which all these views are correct (see Lysak and Song, 2003) to the extent that they explain different features of observations. Auroral electron acceleration by electric field would be operated by at least two mechanisms: largescale quasi-static potential drop (accounting for the classic inverted-V scenario), and fields associated with wave activity (accounting, in particular, for beams in the particle distributions).

In an attempt to conciliate both aspects, together with Polar observations, Janhunen and Olsson (2000) developed a model based on a large-scale O-shaped potential structure which is associated with plasma waves to produce electron energization. Observations by the Fast spacecraft (Ergun et al., 2002a) have shown that quasi-static parallel electric fields are responsible for electron acceleration, in the upward current region; they are self-consistently supported as oblique double layers (Ergun et al., 2002b). However, in the auroral context, the high dynamical variations of the auroral patterns associated with their small spatial scales (in the km range) would suggest an important part to be played by waves. For about 10 years, observations show the importance of Alfvén waves, and more generally of low frequency waves, in particle acceleration (Louarn et al., 1994; Seyler et al., 1995; Andersson et al., 2002b). The role of Alfvén waves in carrying the input energy flux appears to be prominent and has been studied both above (Keiling et al., 2002, 2003) and in
(Chaston et al., 2002) the acceleration region. It turns out that the Poynting flux associated with high-altitude Alfvén waves is sufficient to power electron acceleration to energies and fluxes required by auroras. A study of the Poynting flux associated with kinetic Alfvén waves, at Freja altitude, showed that it is well directed and large enough to power locally observed accelerated electrons (Volwerk et al., 1996). The way this energy is transferred to particles is a key question which the present article proposes to address.

When one tries to understand how the formation of parallel E-fields can be associated to wave propagation, one realizes that other elements inherent to the medium must be invoked in order to form and maintain such fields. It is indeed the interactions of waves with natural turbulent regions, plasma boundaries (magnetic gradients) or highly inhomogeneous regions (density or temperature gradients) which seem likely to provide efficient electron energization mechanisms. Regarding turbulence, stochastic wave/particle interaction has been advocated (Bryant et al., 1991), in auroral and other contexts, with a process equivalent to the Fermi acceleration mechanism. In solar context, Heyvaerts and Priest (1983) have developed the phase mixing concept to account for coronal heating in regions where magnetic gradients are large (see also Ruderman et al., 1999). It is in an equivalent context that we propose here to investigate the complex interaction of an AW with a highly structured plasma, like the one encountered in the auroral zone. Recent analytical work on kinetic AW in inhomogeneous plasma has been conducted by Baronia and Tiwari (2000) in a different geometry than presented here, though; they report that the density inhomogeneity is the fundamental source of free energy for the growth of the kinetic AW, and associated field-aligned currents. Simulations in the ionosphere context (Noël et al., 2000) have shown how parallel electric fields are formed on density gradients and velocity shears when self-consistent feedback of the ionosphere is taken into account. Studying the substorm growth phase, Le Contel et al. (2000) developed a kinetic approach in which a parallel electric field grows proportionally to the pressure gradient. Experimental studies conducted with the Large Plasma Device (Gekelman et al., 2001, and references therein) also investigated the Alfvén wave/inhomogeneity interaction: they reveal that shear Alfvén waves which result from current filaments at scales of several electron skin depths have a parallel electric field. Finally, recent observations of the Cluster mission (Marklund et al., 2001) seem to support the same line: increased field-aligned current and electric field are observed on three satellites which encountered a density gradient region, whereas the fourth satellite, embedded in a plasma with uniform density, exhibits no variation on these two quantities.

Our model of the formation of parallel electric fields associated with AW propagating in density gradients has been initially described in Génot et al. (1999), using the cold plasma model. The basics of parallel field formation and subsequent particle acceleration were tested with self-consistent PIC simulations in Génot et al. (2000), whereas Génot et 
al. (2001) focused on the long-term evolution of the system. This model was able from simple, initial ingredients (an inhomogeneous plasma and low-frequency electromagnetic waves), to reproduce many features observed in auroral plasmas. However, although the chain of individual processes was well understood, the model remained on a descriptive level without further investigation of the physics at work. Recently, simulation works (Silberstein and Otani, 1994; Mandrake et al., 2000; Miyake et al., 2000; Omura et al., 2001; Newman et al., 2001; Umeda et al., 2002) have re-emphasized the potential importance of nonlinear electrostatic structures in the particle acceleration. As these structures appear naturally in our simulation system, we revisit their generation process in the light of the above cited works and more particularly, we address the question of the regime of parameters which favors the formation of the different structures.

Another key mechanism presented here is the energy cascade toward small length scales which seems to correspond to a conversion from an electromagnetic to an electrostatic regime. We thus address the question of the way energy redistribution occurs when a plasma departs from an equilibrium state. We investigate the development of the various instabilities seen in the system using the program WHAMP. Depending on the initial AW amplitude, two instabilities are likely to play a role in the energy cascade: the Buneman instability and the beam-plasma instability, which is the major cause of small-scale structure formation. We use a wavelet analysis to study the energy scale conversion. We classify the small-scale structures and distinguish: 1) unipolar structures, termed weak double layers in the auroral and solar wind contexts (an accumulation of such structures may be an efficient acceleration mechanism), from 2) dipolar structures, termed electron holes because of their shape in phase space. These electron holes are responsible for particle trapping and we present, in the Appendix, an analytical analysis which is compared to simulation results.

In Sect. 2 we present our model. In Sect. 3 we give an overview of the evolution of the system, and we analyze the growth of the parallel electric field and electron acceleration in Sect. 4. Section 5 is devoted to an analysis of the Buneman instability, and Sect. 6 presents the beam-plasma instability, the subsequent formation of small-scales and the different electrostatic structures. In the discussion, Sect. 7, we review the energy cascade process, and in the conclusion we propose some possible plasma environments where the model may be applied.

\section{Model}

As our aim is to investigate how small spatial scales associated with density inhomogeneities perpendicular to the magnetic field modify the AW propagation, the minimal requirement is to use a 2-D model. We developed an implicit 2.5-D (2-D space, 3-D field and velocity components) electromagnetic gyrokinetic code which uses a guiding centre descrip- tion for the electron dynamics while it retains the full ion dynamics. This enables one to save considerable computing time, a precious point for simulating low frequency phenomena. Nevertheless, phenomena of frequencies larger than the electron cyclotron frequency are not described in this treatment. The time step of the simulation can be larger than the electron gyroperiod without incidence on the validity of the results, if the phenomena have characteristic scales longer than the electron gyroperiod. It enables one to follow the full non-linear evolution of the simulated system down to electron inertial length or ion Larmor radius scales. The basics of this code, which uses parallel coding, is described by Mottez et al. (1998). A detailed discussion on its application to auroral physics may be found in Génot et al. (2000), whereas the introductory discussion by Génot et al. (2001) focused on the difficulty in comparing simulation parameters and natural ones. In the following we summarize the important points relevant to the present study.

In the code, the time, the velocity, the mass, and the charge are, respectively, normalized to $\omega_{p e}^{-1}, c, m_{e}$, and $e$, the electron charge. The ambient magnetic field amplitude is given by the value of the ratio $\omega_{c e} / \omega_{p e}$. The electric field is normalized to $\omega_{p e} c m_{e} / e$. The mass ratio $m_{i} / m_{e}$ is reduced to 100 , in order to follow both electron and ion dynamics within a reasonable computation time.

We use the spatial configuration given in Fig. 1. The simulation box is a grid of $2048 \times 64$ cells having an individual size of $0.01\left(c / \omega_{p e}\right)^{2}$. The box thus physically corresponds to $\sim 1000 \mathrm{~km}$ in the parallel direction and $\sim 40 \mathrm{~km}$ in the transverse direction, assuming a density of $1 \mathrm{~cm}^{-3}$. The system is periodic in both directions. Since the length of a grid cell must be of the order or smaller than a Debye length $\lambda_{D}$, the electron temperature cannot be smaller than a limit value. In our simulations the electron thermal velocity, $v_{\text {the }}$, is 0.1 in normalized unit, and ten times larger than the ion thermal velocity, $v_{t h i}$ (which implies $T_{e}=T_{i}$ ). The density in the middle of the cavity is typically four times less than the one on both sides. The density variations perpendicular to the magnetic field occur on about one electron inertial length. The simulations presented here describe the impulsive response of this static inhomogeneous plasma to an incoming electromagnetic flux associated with AW. The Alfvén wave initialized in the system propagates from the left to the right and has no associated parallel electric field. In the following, we describe the evolution of the system on several Alfvén periods for perturbations up to $\delta B / B=0.05$. By varying this initial amplitude, it will be possible to investigate the non-linear response of the plasma and the instability triggering. The Alfvén wavelength, equal to the size of the box, is sufficiently long to restrict the propagation to a domain of a small wave number, where the Alfvén wave dispersion is practically negligible since the ion cyclotron to Alfvén frequency ratio is $\omega_{c i} / \omega_{A}=4$. The normalized Alfvén velocity is 0.4 which gives a plasma to magnetic pressure ratio $\beta=4 \frac{m_{e}}{m_{i}}\left(\frac{v_{\text {the }}}{v_{A}}\right)^{2}=0.0025$. The total number of particles is $\sim 5 \times 10^{6}$. 


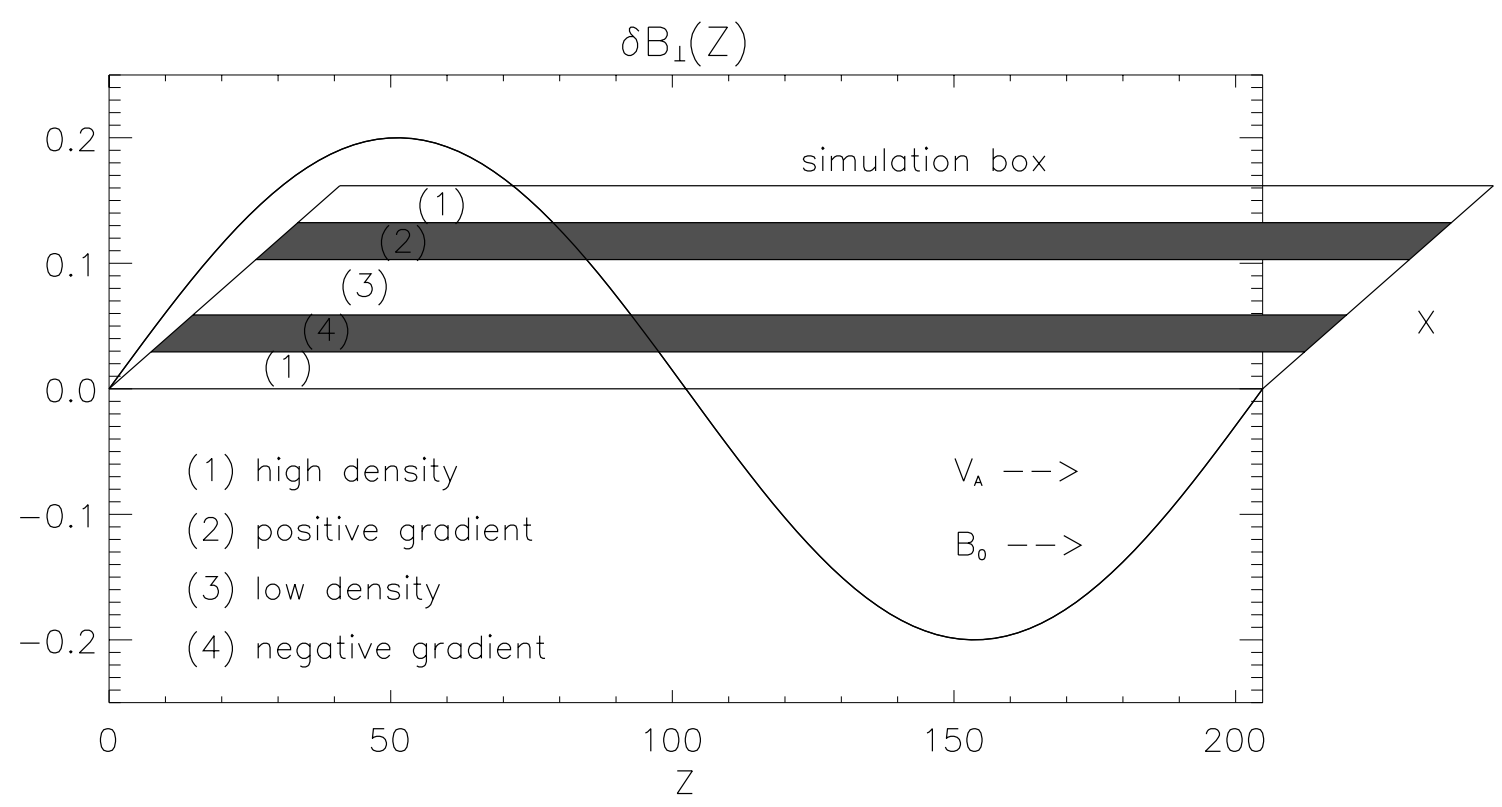

Fig. 1. Configuration of the simulation. The Alfvén wave propagates in the $Z$ direction and the density gradients are in the $X$ direction.

\section{General overview of the simulation results}

We present here a summary of the evolution observed as the wave propagates. The rapid evolution of the system may be tracked on the parallel component of the electric field. Starting from zero value (initial $k=k_{z}=k_{\|}$, i.e. $E_{z}=E_{\|}=0$ ), the behavior of this component may be schematically described in the following phases: a linear growth phase with possible development of instabilities, a nonlinear phase leading to small-scale structures and finally a recovery phase. These phases, observed and labeled in Fig. 2 and Fig. 3, are quickly described below and detailed in the following sections of the paper.

The plots in Fig. 2 and Fig. 3 display the parallel component of the electric field on the density gradients of the cavity, for $\delta B / B=0.05$ and $\delta B / B=0.025$, respectively. We use the periodicity of the model to present a double-sized box along the magnetic field which eases the visualization of important features. We also describe the action of the parallel electric field on the particles (a history of the particle energy is presented later).

- During an initial phase, a parallel electric field is created in the density gradient regions, on spatial scales of the order of the incoming AW wavelength $(\lambda=204.8)$. This initial phase corresponds to $t<350$ in Fig. 2 and to $t<500$ in Fig. 3 (see label 1). This long range $E_{\|}$ reaches maximum amplitude of, respectively, $\sim 0.02 E_{\perp}$ and $\sim 0.01 E_{\perp}$. This is the order of magnitude obtained for an inertial Alfvén Wave with $k_{\perp} c / \omega_{p e} \sim 1$ and $\lambda_{\perp} / \lambda_{\|} \sim 0.03$.

- Due to the direction of propagation of the incoming AW (here toward $z>0$ ), a dissymmetry appears in the energy exchange with the electron population since the positive $z$-traveling electrons are preferentially accelerated. Therefore, the maximum acceleration occurs in regions where the parallel electric field is opposite to the direction of the incoming wave propagation.

- For sufficiently large wave amplitudes, a first instability is triggered during this initial stage. Its signature on the parallel electric field can be seen as backward propagating, small wavelength waves in Fig. 2, starting at $t \sim 350$ (see label 2, the corresponding stripes have a negative slope in this position-time representation, indicating a negative velocity). Nothing comparable can be seen in Fig. 3.

- As a result of the particle acceleration, runaway electrons escape from the region where they have been initially accelerated and create a beam, downward from the acceleration region. The formation of the electron beam, which has been systematically observed in the simulations, marks the end of the initial linear stage.

- Indeed, this beam becomes unstable. An electrostatic beam-plasma instability suddenly takes place and leads to a fast re-organization of the parallel electric field into small-scale nonlinear structures. This is observed from $t=450$ and from $t=600$ in Fig. 2 and Fig. 3, respectively, (see label 3).

- Different types of nonlinear structures form. Some of them keep a modest amplitude, they do not present a reversal of the electric field and can be assimilated to weak double layers. Stronger ones, associated with the reversal of the field, are also observed and present similarities with electron holes in which particles are trapped. 


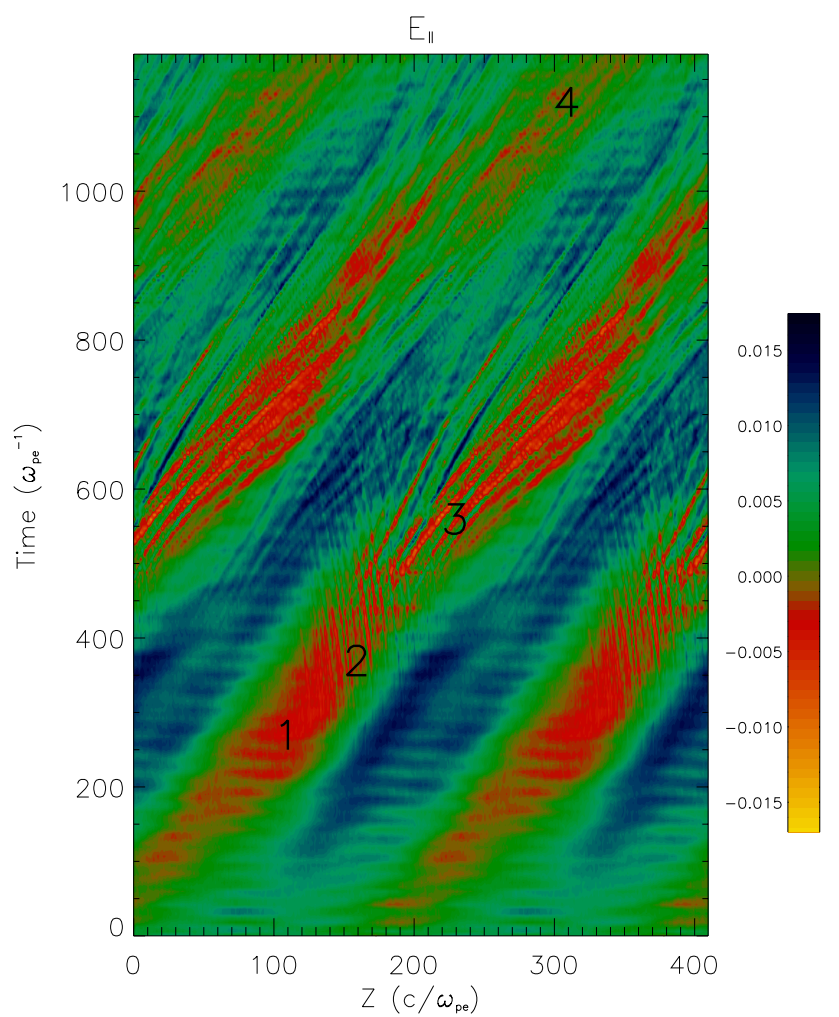

Fig. 2. Time evolution of the parallel electric field averaged on the density gradient (large amplitude case, $\delta B / B=0.05$ ). Two periodic simulation boxes have been piled up to reveal the formation of small-scale structures. Labels 1-4 correspond to the predominance of: the large-scale field (1), the Buneman instability (2), the beam-plasma instability (3), the large-scale field again (4).

- The system recovers from its previous nonlinear phase dominated by small-scale structures. The large-scale one (box sized) progressively returns to be the predominant structure of the parallel electric field (from $t=1000$ ) (see label 4).

In the following sections we shall focus on each physical process described above.

\section{Linear formation of the parallel electric field}

We recall that the initialization is such that the parallel component of the electric field is initially zero. In the simple cold plasma model studied in Génot et al. (1999), it has been shown that the polarization drift induced on the density gradient plays a leading role in the formation of a space charge and the subsequent parallel electric field. Let us here estimate the order of magnitude of this drift, how it compares to the $\boldsymbol{E} \times \boldsymbol{B}$-drift, and finally, what parallel electric field may be expected. The ion polarization current can be written:

$j_{x}=\frac{m_{i} n_{i}}{B^{2}} \frac{\partial E_{x}}{\partial t}=n_{i} e v_{p}$

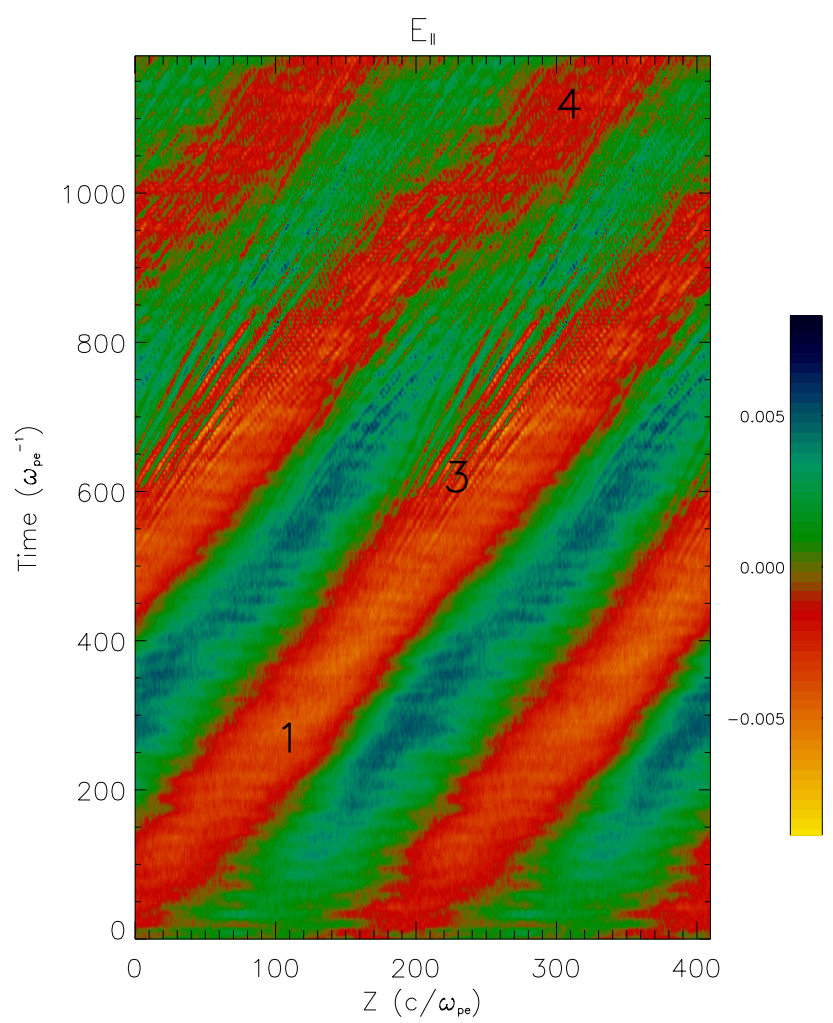

Fig. 3. Similar to Fig. 2 for the low amplitude case $\delta B / B=0.025$. The Buneman instability (label 2 in Fig. 2) is not triggered.

then, for sinusoidal perturbations ( $v_{p}$ is the polarization velocity),

$v_{p}=\frac{\omega_{A}}{\omega_{c i}} i \frac{E_{x}}{B}$.

The $\boldsymbol{E} \times \boldsymbol{B}$ velocity in the $x$ direction is given by

$v_{\boldsymbol{E} \times \boldsymbol{B}}=\frac{E_{y} B_{z}}{B^{2}} \simeq \frac{E_{y}}{B}$.

For the Alfvén wave, we have $E_{x} \simeq E_{y}$, therefore $v_{\boldsymbol{E} \times \boldsymbol{B}} / v_{p}=\omega_{c i} / \omega_{A}$; this ratio is greater than 3 in the simulation, and even for a large amplitude wave, this yields a polarization velocity smaller than the ion thermal speed. However, since the $\boldsymbol{E} \times \boldsymbol{B}$-drift is not mass dependent, it is the same for electrons and ions and does not lead to electrostatic effects. On the contrary, the polarization drift is proportional to the mass. It is therefore negligible for electrons and only significantly displaces ions. This causes the formation of a space charge sufficient to build a large-scale parallel electric field, as shown below.

Let us now estimate the behaviour of the electric field from the Poisson equation assuming that the parallel electric field remains zero. From the Poisson equation we get in normalized units,

$\left|E_{x}\right|_{\max }=\left(1-\frac{n_{\min }}{n_{\max }}\right)(\Delta x)_{\max }$, 


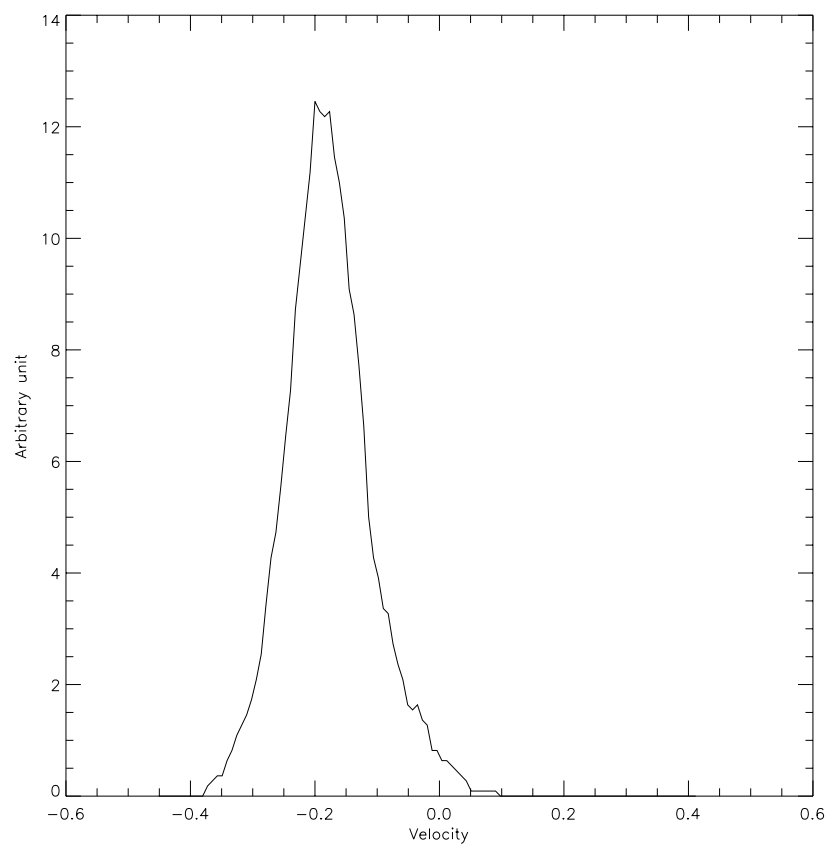

Fig. 4. Electron velocity distribution function leading to the Buneman instability at $\omega_{p e} t=320$ and $Z=162 c / \omega_{p e}$ (below label 2 in Fig. 2) in the density gradient region.

where $(\Delta x)_{\max }$ is the largest spatial separation of the ions from the electrons on the density gradient. Typically: $(\Delta x)_{\max }=2 v_{p_{\max }} / \omega_{A} \simeq 1$. We assume a factor 4 in the decrease of the density, as in simulations, and $v_{p}=v_{p_{\max }} \sin \left(k z-\omega_{A} t\right)$, with $v_{p_{\max }}=0.006$ which corresponds to the typical value calculated from Eq. (2). Then $\left|E_{x}\right|_{\text {max }} \simeq 0.75$. This means that, in the absence of the parallel electric field, the electrostatic perturbation induced by the polarization drift driven space charge would be one order of magnitude larger than the Alfvénic perturbation ( $E_{A W}=v_{A} \delta B=0.08$ ). Two conclusions can be drawn from this calculation. First, the parallel electric field clearly plays an important role in restoring electro-neutrality. As the low inertia electrons are the fastest moving particles, the parallel direction is the preferred one for the setting of the neutralization electric field. The electron acceleration is thus a direct consequence of the formation of small electrical non-neutralities on the density gradients. Second, from $(\Delta x)_{\text {max }} \simeq 1$ we deduce that the space charge extends up to 10 Debye lengths across the magnetic field on the density gradient location $\left(c / \omega_{p e} \lambda_{D}=10\right)$. This is an estimate of the width of the acceleration region. Actually, this scale corresponds to what is expected from a direct measure on particles: for instance, for the simulation presented in Fig. 3a of Génot et al. (2000), which presents comparable conditions to the present simulations, the space charge extends on $\sim 5$ Debye lengths. In the parallel direction, the length is half the Alfvén wavelength (the distance on which the polarization drift keeps the same sign).
In Fig. 2 we notice that the time scale of the formation of the parallel electric field component is very short. Is it consistent with the initial linear theory? From Eq. (2) one sees that from the beginning of the wave propagation, the polarization drift is active to separate ions from electrons. The maximum drift is obtained after half the Alfvén period which corresponds to $\sim 250 \omega_{p e}^{-1}$. Being a plasma response to electric non-neutrality, the parallel electric field grows on a time scale given by the plasma period. Therefore, the field response to the drift is almost instantaneous and the field formation is completely driven by the growth of the drift which directly generates the electric non-neutrality. Indeed, in Fig. 2 we notice that the maximum of the parallel electric field is obtained around $250 \omega_{p e}^{-1}$. Starting from $t=0$, we can follow its growth both in amplitude and length scale: at $t=50 \omega_{p e}^{-1}$ the parallel extension is $\Delta z \simeq 50 \mathrm{c} / \omega_{p e}$, whereas at $t=250 \omega_{p e}^{-1}$ we measure $\Delta z \simeq 100 c / \omega_{p e}$ (half the Alfvén wavelength). These regions of parallel fields are centred on the regions where the maximum drift is expected to occur and thus, where the electric space charge begins forming.

The parallel electric field whose formation mechanism is described here is directly responsible for most of the particle acceleration. However, it must be stressed that the situation is not equivalent to electrostatic acceleration. If we suppose a static parallel electric field of the form $E_{\|}=-A \sin k z$, an electron with no initial velocity would be accelerated to $0.4 c$ (the wave velocity) over half a wavelength for $A \simeq 0.0012$ in code normalized unit. This value is consistent with the maximum $E_{\|}$reached after growth. However, the parallel field in the simulation is not static, which greatly modifies the characteristics of the acceleration. In particular, due to the large propagation velocity of the wave the acceleration of thermal particles is limited. If we consider the regions of negative parallel field, electrons with negative velocities will be very little decelerated during their encounter with the field, while electrons with small positive velocities will be accelerated but quickly overtaken by the field. Only originally fast electrons, which almost travel with the wave and see a static field, will be sufficiently accelerated to form a suprathermal population which is later seen as a beam. Therefore, the energy gained by electrons is a function of their initial velocity, as well as the amplitude and the velocity of the wave. We shall come back to this question in Sect. 7.

\section{Early stage instability: the Buneman instability}

Let us present this instability and the way it is sensitive to the initial conditions: adient size $\left(1.9 \omega_{p e} / c\right)$ and $\delta B / B=0.025$. On the $(z, t)$ plane in Fig. 2, the instability manifests itself as small-scale structures of the parallel electric field with a typical wavelength of a few $c / \omega_{p e}$. This perturbation travels backwards (towards $z<0$ ) and is seen as early as $\omega_{p e}^{-1} t=260$ for $z=125 c / \omega_{p e}$. In Fig. 3 the instability could be expected to appear later because of the wave lower amplitude. In fact, it is not triggered at all, which suggests that a threshold exists above which the instability develops. 

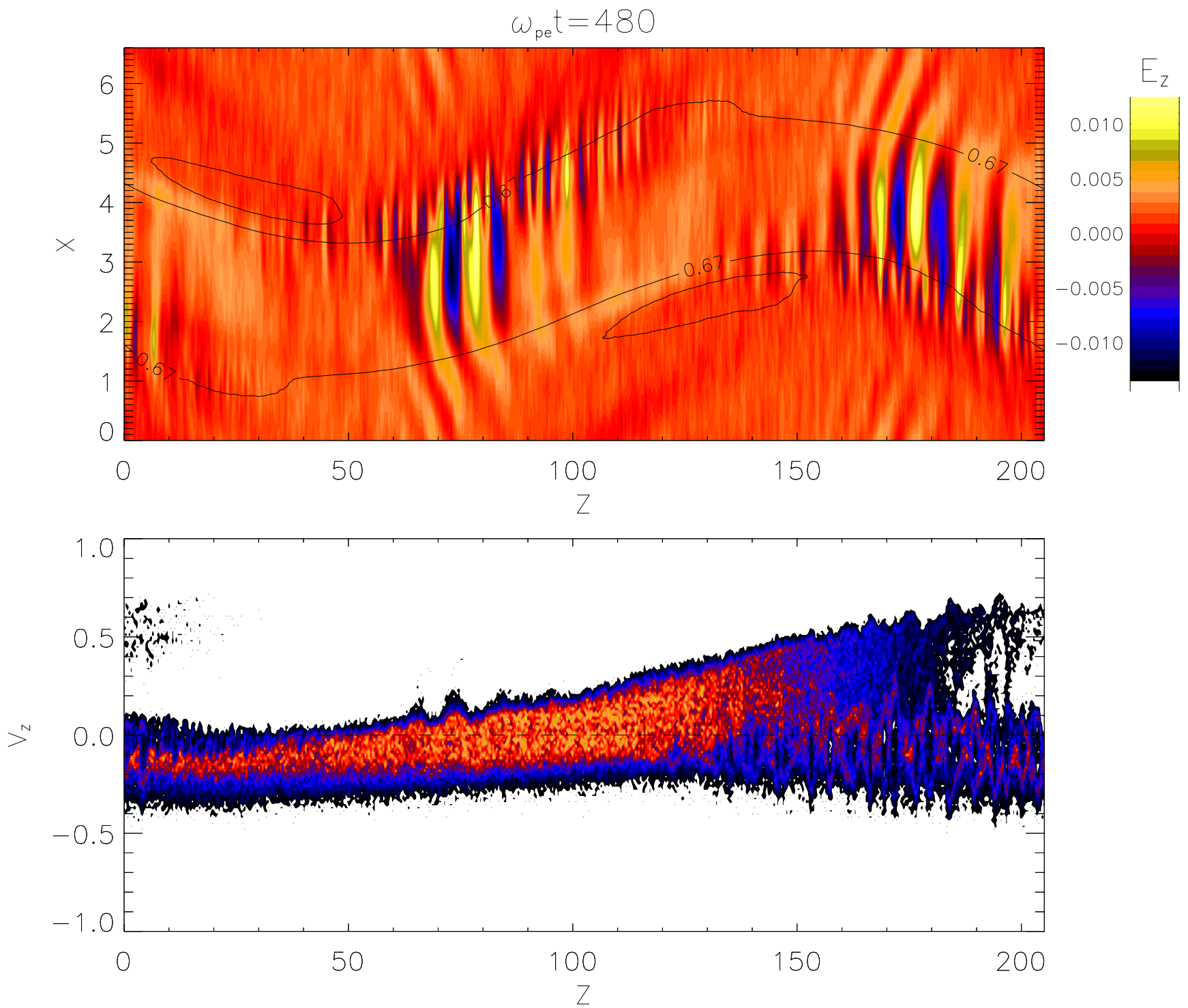

Fig. 5. Parallel electric field and parallel electron phase space at $\omega_{p e} t=480(\delta B / B=0.05)$. The electric field is shown in the 2-D simulation box with colour-coded amplitude and with density contours overlaid; the " 0.67 " line coarsely defines the middle of the region of density variation along the $X$ direction. The bottom panel is the electron velocity distribution function in the parallel velocity direction and along the magnetic field. Each dot corresponds to a particle in this coordinate space. Only electrons located in the lower gradient region have been selected.

This particular signature of the electric field is not seen on the magnetic field components: the perturbation is electrostatic and appears to be the consequence of a large disturbance on the electron distributions. Indeed, the electrons gained large amounts of energy from their interaction with the parallel electric field and, locally on the gradient, this energy can be such that the whole electron distribution is shifted in velocity space. This is shown for the large amplitude case in Fig. 4, in a region where the electric field is positive. The velocity drift corresponds to twice the thermal velocity. Such a situation is likely to trigger the Buneman instability. For the lower amplitude case, the drift is also present in the equivalent region but is not larger than the thermal velocity.
To validate the previous scenario, we investigate the conditions mandatory for the triggering of the Buneman instability in the regime of parameters of the simulation. We perform a numerical analysis of the Maxwell-Vlasov linear equations with the program WHAMP (Rönnmark, 1982). We use particle mass ratios analogous to those of the simulations. Although our model is not homogeneous, as long as the relevant perpendicular wavelengths of the unstable waves are small in comparison with the inhomogeneity length scale, the homogeneous approximation is adequate. As a matter of fact, we observed that the condition $v_{d r i f t} / v_{\text {the }}=2$ (as seen in Fig. 4) leads to a positive growth rate with WHAMP, whereas $v_{\text {drift }} / v_{\text {the }}=1$ or less leads to a stable situation; $v_{\text {drift }}$ is the velocity drift of the electron distribution function. For 


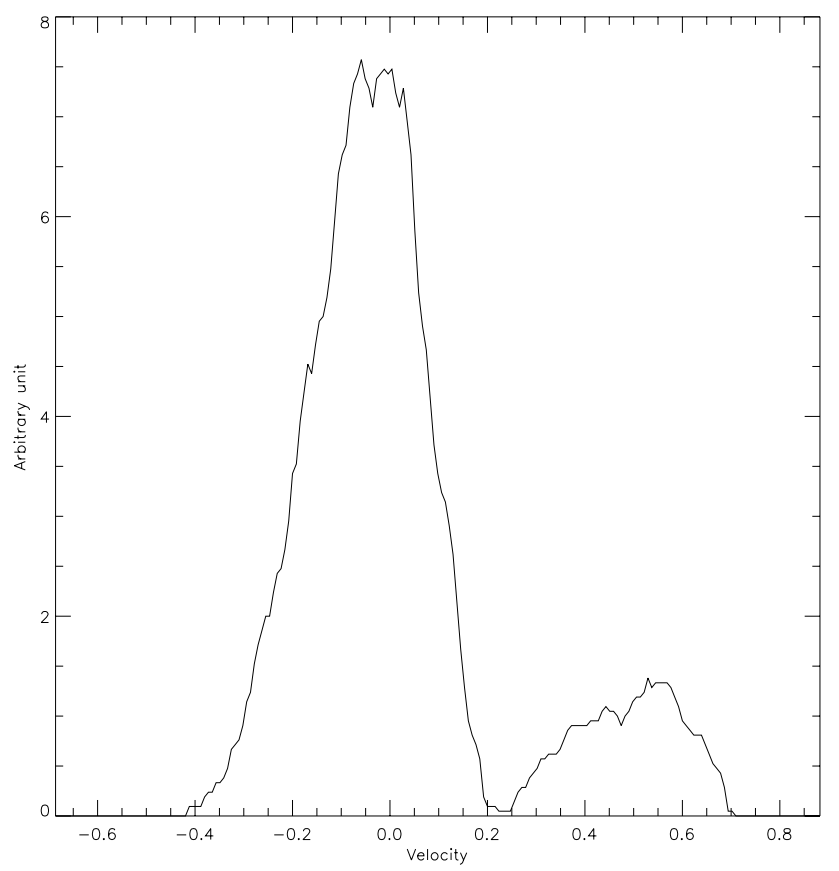

Fig. 6. Electron velocity distribution function leading to the beamplasma instability at $\omega_{p e} t=480$ and $Z=188 c / \omega_{p e}$ (between labels 2 and 3 on Fig. 2) in the density gradient region.

comparison, the condition $v_{d r i f t} / v_{\text {the }}=2$ leads to stability for a plasma with higher $\omega_{c e} / \omega_{p e}$ than in the simulation (which corresponds to more magnetized or less dense situations). Also, we note that in the theoretical expression stating the condition for marginal stability on the wave number, a particle mass factor is involved:

$k v_{0}=\omega_{p e}\left(1+\left(\frac{m_{e}}{m_{i}}\right)^{1 / 3}\right)^{3 / 2}$

Its value is only $\sim 20 \%$ higher in the simulation than in reality: this is an indication that the comparison reality/simulation may well apply. The Buneman frequency is such that $\omega_{b u n} / k \propto v_{0}$, and the drifted population is found in a positive electric field region, therefore $v_{0}<0$ (as seen in Fig. 4) and the electrostatic waves associated with the Buneman instability are backward propagating as actually observed. The maximum growth rate computed from WHAMP corresponds to a growth time of $\sim 42 \omega_{p e}^{-1}$. This is consistent with the time between the first appearance of the Buneman instability perturbations and the time when they saturate and reach their maximum (see the growth of the perturbation for $125<z<200$ and $260<\omega_{p e} t<425$ in Fig. 2). We calculate $\omega_{\text {bun }}=0.085 \omega_{\text {pe }}$ which is in the range of the ion plasma frequency: this indicates that the waves are ion acoustic waves. The electrostatic structures associated with the Buneman instability are sufficiently stable in time (more than $300 \omega_{p e}^{-1}$ ) and reach amplitudes $\left(E_{\|} \simeq 0.002\right)$ large enough to trap particles. This is seen in the electron phase space in Fig. 5: for $140<z<200$ some round-shaped trapping structures are centred on negative velocities $(\sim-0.1)$. Simultaneously in the same position range, the parallel electric field shows a succession of positive and negative peaks just below the density contour line labeled " 0.67 ", which corresponds to the middle of the gradient region. These structures are similar in shape to the larger structures described in the following section. A plot of the electron phase space for the low amplitude case would not show the trapping structures, as it does not show instability in Fig. 3. We thus conclude that for sufficiently intense $\mathrm{AW}$, able to trigger a drift velocity larger than $2 v_{\text {the }}$, the Buneman instability of ion acoustic waves explains the backward propagating small-scale structures of the parallel electric field.

\section{Scale transition through the beam-plasma instability}

6.1 The beam-plasma instability and associated field structures

In the region of minimum parallel electric field, a part of electrons are accelerated to velocities larger than the wave phase velocity and form a beam. This is shown in Fig. 6, where the beam velocity distribution peaks at $\sim 0.55$. This beam becomes unstable with respect to the plasma and gives rise to the beam-plasma instability. The signature of the instability is clearly seen on the parallel electric field component (see Fig. 2) which displays narrow electrostatic structures in which particles may be trapped. We identify two kinds of structures with different scales and amplitudes. Both are different from the ones associated with the Buneman instability and previously described.

- Bipolar structures: the electric potential difference experienced by an electron traveling across these structures is zero. Their typical scale is about 100 Debye length and their amplitude is larger than the large-scale parallel electric field. They are termed electron holes. The bulk of them travels at a velocity near the Alfvén velocity but the velocity dispersion is not negligible, leading to velocities between 0.3 and 0.6.

- Weak double layers (DL): smaller in length scale and amplitude, these unipolar structures present a net potential difference at their sides. Their typical electric potential amplitude is such that $e \Delta \Phi / k T_{\text {the }} \leq 2$. They have the same propagation properties as the bipolar structures and seem to correspond to a non-symmetric decay of these bipolar structures. They are associated with a reorganization of the field and their lifetime is shorter than the bipolar structures' stability time.

Figure 7 displays examples of these structures. The dotted line corresponds to an integration of the electric field, and is therefore proportional to the electric potential. The symmetric bipolar structure is seen as an unipolar electric potential with no net difference at its sides. Both structures can be located in the top panel of Fig. 8: the DL is observed at $z \simeq 148$ and $x \simeq 1.5$, inside the lower gradient, whereas the bipolar structure is at $z \simeq 125$ and $x \simeq 3$. 

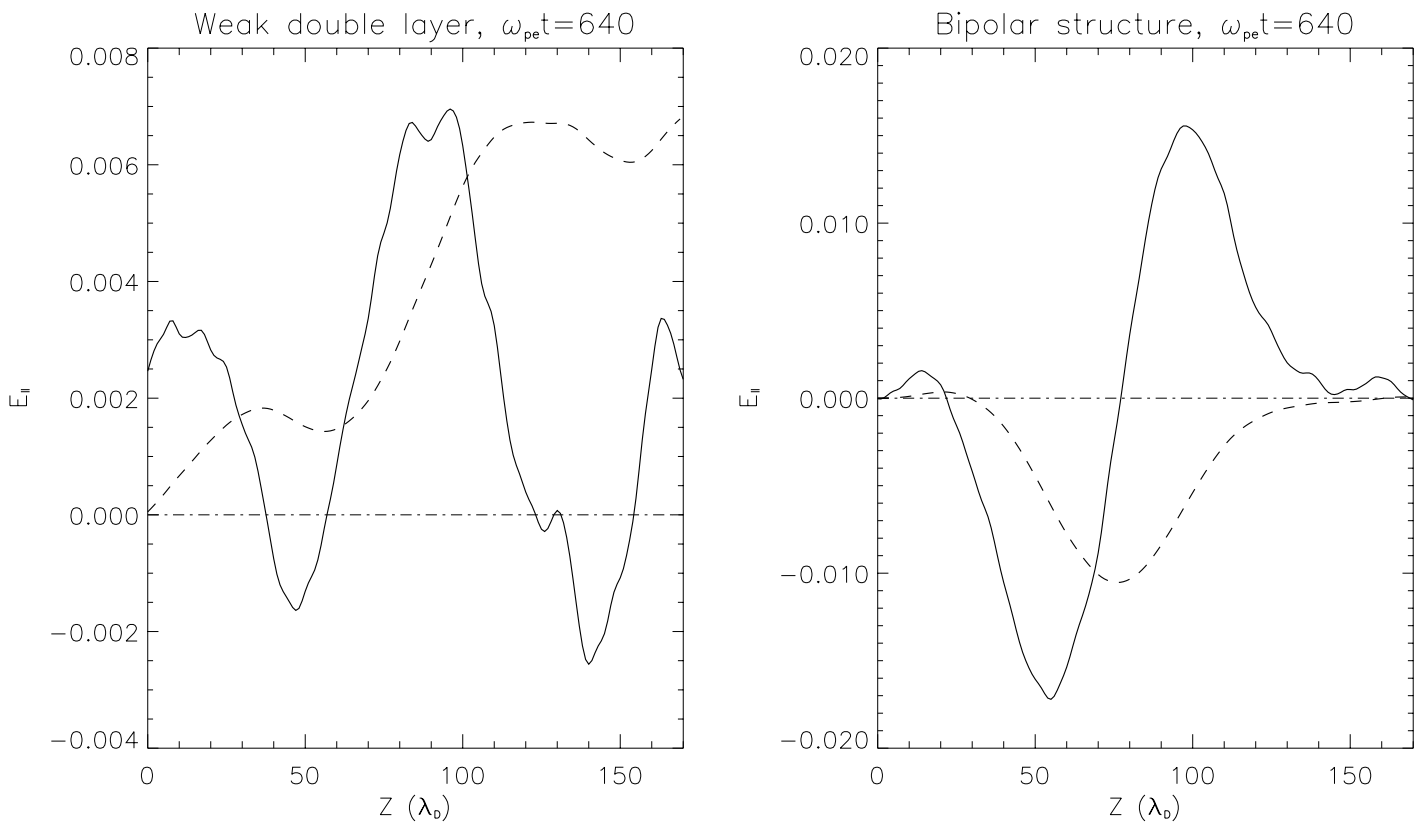

Fig. 7. Two different electric field structures at the same time (solid line). The spatial extension along the magnetic field is similar. The dashed line on both plots is proportional to the integrated electric field, i.e. the electric potential: it is non-null for the weak double layer (left panel), whereas it is zero for the symmetric bipolar structure (right panel).

These double layers are similar in amplitude to those commonly observed in the auroral zone $\left(e \Delta \Phi / k T_{\text {the }} \simeq 1\right)$. They are much larger than those observed in the solar wind $\left(e \Delta \Phi / k T_{\text {the }} \simeq 10^{-3}\right)$, but smaller than those recently reported by FAST $\left(e \Delta \Phi / k T_{\text {the }} \geq 20\right)$ and discussed later (Sect. 6.3).

Investigating the spatial scales of the electron holes (in Fig. 8 and equivalent views at other times) reveals a parallel to perpendicular length ratio of $\sim 2-4$. Different analytical studies supported by observations (Franz et al., 2000; Singh, 2000), however, expect a roughly spherical shape $\left(L_{\|} / L_{\perp} \simeq 1\right)$ for plasma with $\omega_{c e} / \omega_{p e}>1$, as in the simulations. However, these studies focus on uniform plasmas, while electron holes in our simulations appear in the inhomogeneous region. The density gradient may confine the structure spread in the perpendicular direction. From the middle of the gradient, we observe in Fig. 8 that the electron holes extend further towards the lower density side, whereas the higher density side is less extended; electric fields are indeed more easily short-circuited in a denser medium. However, a recent analytical study in cylindrical geometry (Chen and Parks, 2002) showed that for a fixed potential amplitude and perpendicular scale size, the parallel scale size has a lower bound but no upper bound. Also, for a fixed potential amplitude the perpendicular scale size has a lower bound. Therefore, for a fixed potential amplitude the aspect ratio of the electron hole $L_{\|} / L_{\perp}$ can take a broad range of values (both $<1$ and $>1$ ) but there exists a restricted domain where this ratio has to be $>1$ for physical solutions to exist. This analysis allows the situations observed in the simulations.

\subsection{Investigation with WHAMP}

Similarly to what we have done for the Buneman instability, we investigate with WHAMP the plasma instabilities which may develop in the plasma at this stage of the evolution of the system. The distribution functions shown here are computed from all the particles located in the negative gradient region. The local plasma frequency is then smaller than the plasma frequency outside the cavity.

To initialize WHAMP we use the parameters derived from the distribution given in Fig. 6 at $\omega_{p e} t=480: v_{\text {the }}=0.12$, $v_{\text {the-beam }}=0.13, \quad v_{\text {drift }}=-0.05, \quad v_{\text {drift } \text {-beam }}=0.5$, $n_{e-\text { beam }} / n_{e}=0.17$. These values indicate that the plasma is likely to be unstable to the weak beam instability which exists in the regime:

$\frac{v_{\text {the-beam }}}{v_{\text {drift-beam }}} \ll\left(\frac{n_{e-\text { beam }}}{n_{e}}\right)^{1 / 3}$.

This is confirmed by WHAMP: a positive growth rate is found in the range $0<k c / \omega_{p e}<6$ with a maximum growth rate at $k c / \omega_{p e}=3$ which corresponds to wavelengths $\sim 20 \lambda_{D}$ (its value is $\gamma_{\max }=6 \omega_{c i}=0.24 \omega_{p e}$ ). The associated frequency is in the range of the plasma frequency and corresponds to Langmuir waves excited by weak beams. These waves are effectively observed on a dispersion plot obtained by a double Fourier transform of the field given in Fig. 2 (not shown). At the same time, for the low amplitude case, the conditions of the plasma are less in favor of the triggering of the instability, but still a positive growth is found in the same wave number range $\left(v_{\text {the }}=0.1, v_{\text {the-beam }}=0.1\right.$, $\left.v_{\text {drift }}=-0.04, v_{\text {drift-beam }}=0.3, n_{\text {ebeam }} / n_{e}=0.1\right)$. The 

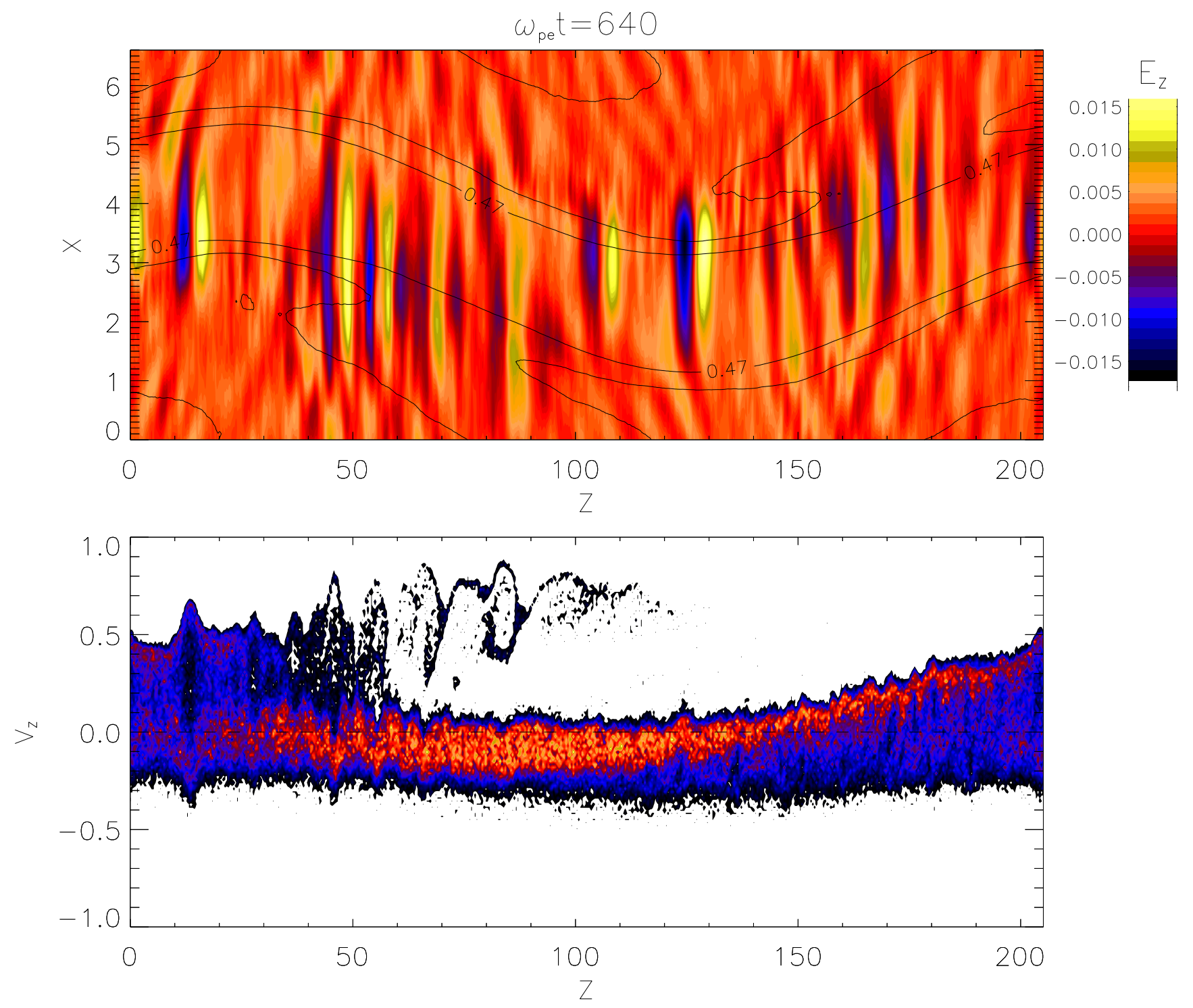

Fig. 8. Similar to Fig. 5 for $\omega_{p e} t=640$. The " 0.47 " line in the upper panel marks the middle of the gradient region.

maximum growth rate is smaller $\left(\gamma_{\max }=3.8 \omega_{c i}=0.15 \omega_{p e}\right)$ and is obtained for a slightly smaller wavelength than in the previous case. However, the associated frequency is still in the range of the plasma frequency. In conclusion, the results of the investigation with WHAMP are consistent with the evolution shown in Fig. 2 and Fig. 3. The lower the initial wave amplitude, the smaller the velocity of the electron beam and the smaller the instability growth rate of the Langmuir waves.

For high wave amplitude, Eq. (6) is fulfilled in the whole region of the negative electric field. In Fig. 2, ones observes the fluctuations associated with the instability from $z=140 c / \omega_{p e}$ to $z=190 c / \omega_{p e}$ at $\omega_{p e} t \simeq 450$. On the contrary, in Fig. 3, the development of these fluctuations is slower and they appear more gradually, first in the region where electrons have experienced the field for the longer time, i.e. on the trailing edge of the negative electric field, and then for larger $z$ as the beam propagates (note the broadening of the small-scale structure location for $550<\omega_{p e} t<800$ ).

\subsection{Comparison with another simulation}

In Newman et al. (2001), the authors investigate the formation of double layers and electrons holes with 1-D simulations in which a beam turns the plasma instable. They show how electron holes form and evolve toward a strong double layer $\left(e \Delta \Phi / k T_{t h e} \geq 20\right)$ whose associated unipolar electric field is similar to those observed with FAST. Their Fig. $1 \mathrm{c}$, which displays the electron phase space $\left(z, v_{z}\right)$, presents features very similar to those seen in our Fig. 8 , in almost the same length scale and velocity range $\left(v / v_{t h e} \sim 5\right.$, $\left.\Delta z \sim 100 \lambda_{D}\right)$. In particular, in front of the electron beam are structures similar to the ones seen for $60<z<120$ in Fig. 8 and zoomed in Fig. 9. What are the differences? First, if 

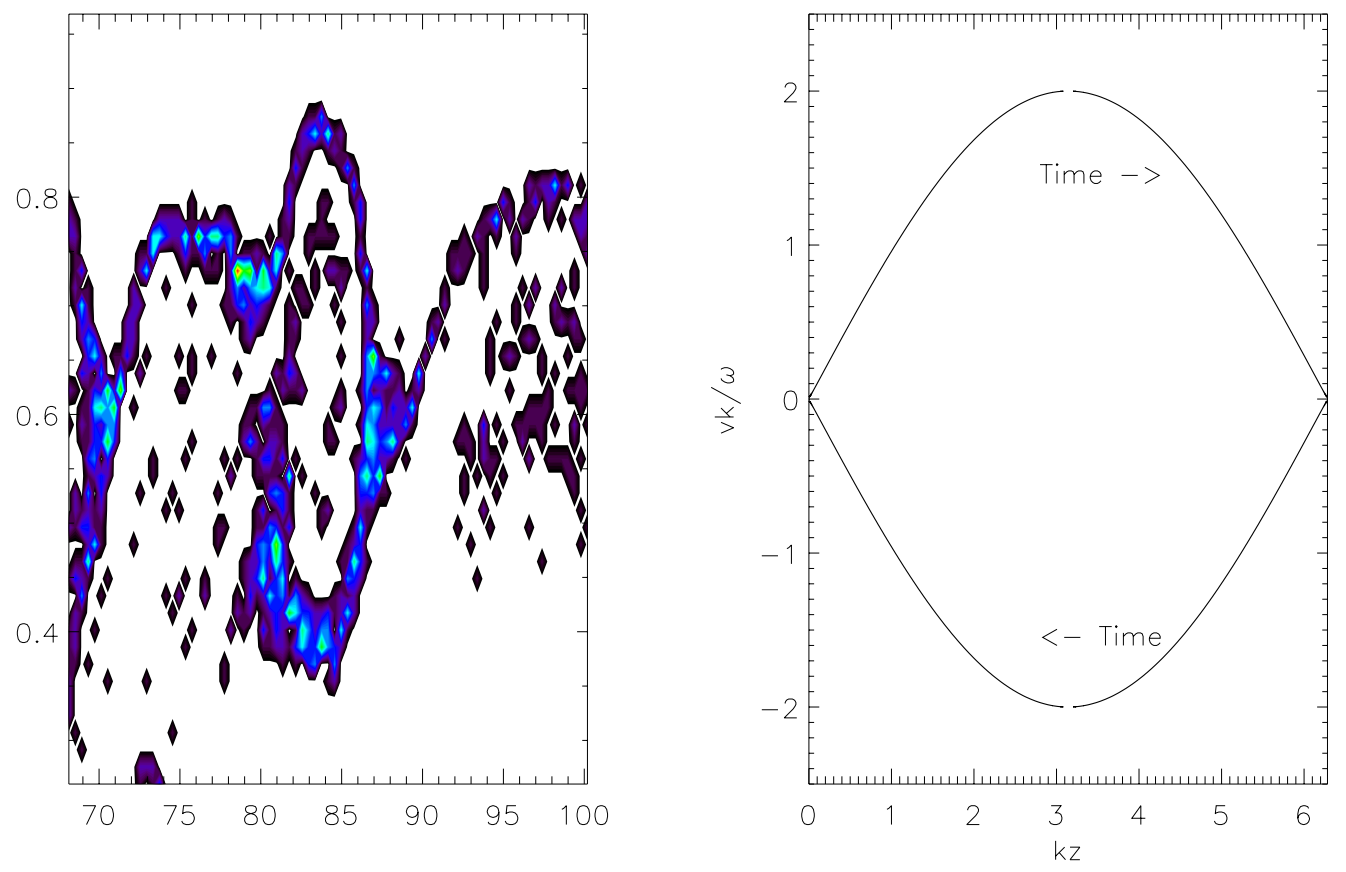

Fig. 9. (Left) zoom on the electron hole observed in the large amplitude case at $\omega_{p e} t=640$ (see Fig. 8), and (right) analytical solution of electron hole from a simple model (see Appendix for details).

we concentrate on this particular structure, the field associated with it in our simulation is clearly dipolar, whereas it is unipolar in Newman et al. (2001). In brief, they have a double layer, we have an electron hole. In our case, we see a closed ring in phase space, whereas their case presents a partial ring. The fact that in our simulations we do not see the evolution toward a strong double layer may be linked to the free energy source: the electron beam. Indeed, in Newman et al. (2001) the beam is sustained during the whole simulation and able to supply free energy to the evolving structures (electron holes and then strong double layers), whereas in our case, the beam is a transient phenomenon which vanishes as acceleration stops and electrons becomes trapped (see Sect. 7 for a further analysis of this evolution). Our simulations follow Newman et al. (2001) in their conclusion that "the presence of a strong DL might be sufficient but not necessary for electron-hole generation". We expect that a longer acceleration phase (related to a combination of longer wavelength, increased wave amplitude, and decreased gradient size) is bound to lead to a more persistent beam, and may give rise to the formation of a strong DL.

\subsection{Formation of small-scale structures}

A way to study the evolution of the system is to present the characteristic length scales associated with the parallel electric field. Indeed, changes in this quantity reveal adequately the change in the system regime (linear to nonlinear, electromagnetic to electrostatic). We perform a wavelet analysis of the parallel electric field in the density gradient region as a function of the position parallel to the magnetic field and at different times. The contours of the colour-coded wavelet power spectra are then plotted as a function of position and scale. We chose a Morlet wavelet; actually, the choice of the function is not critical as long as one is interested in wavelet power spectra (Torrence and Compo, 1998). A different function would lead to the same positions and scales for the peaks in the spectra, and this is precisely these positions and scales which we are interested in. We present the large amplitude case for which each scale regime is clearly distinguishable. The results are displayed in Fig. 10. For each panel, the upper part is the parallel electric field averaged on the negative density gradient and the bottom part is the result of the wavelet analysis. At $\omega_{p e} t=160$, the electric field is mainly organized on the large-scale, of the order of the size of the simulation box, 204.8c/ $\omega_{p e}$, which explains the red line in the power spectrum at this scale. The rest of the spectrum is noise around the electron skin depth scale. Later, at $\omega_{p e} t=280$, the large-scale is still present but one sees the formation of localized structures on a few $c / \omega_{p e}$ : this corresponds to the fine structures associated with the Buneman instability. A detailed analysis at close successive times reveals that these structures propagate toward the negative $z$. This is not easy to detect as the negative velocity is small (the stripes are almost vertical in Fig. 2, near label 3). For instance, note how between $\omega_{p e} t=280$ and $\omega_{p e} t=344$, the power maximum between scale 1 and 10 has extended toward the left whereas its right edge remained stationary. For later times, there is a quick transition to another kind of structures: they are the ones associated with the beamplasma instability; they are more extended along the magnetic field direction (mostly larger than $10 c / \omega_{p e}$ ), and their power almost completely overtakes the large-scale electric 

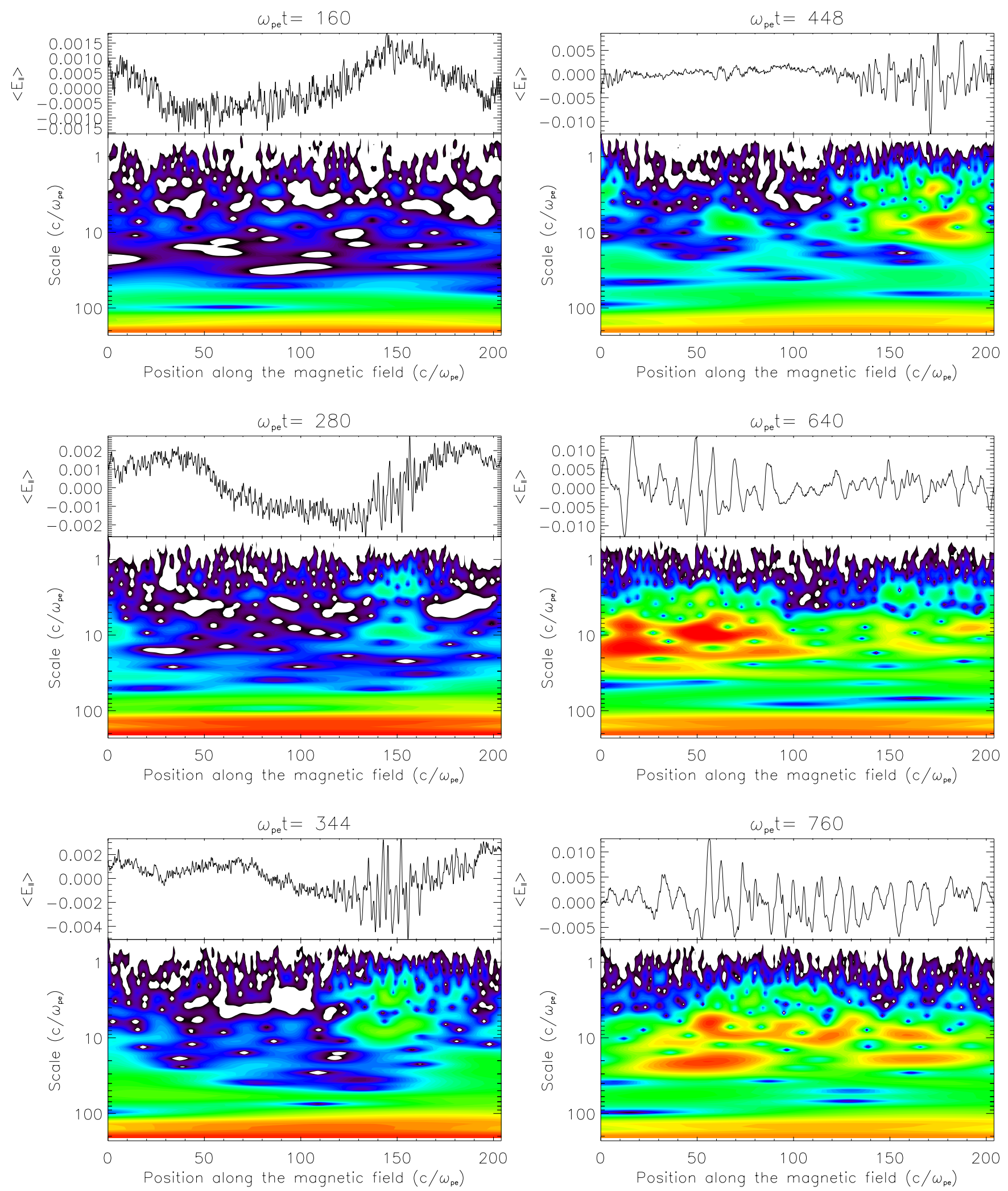

Fig. 10. Wavelet analysis of the parallel electric field at different times of the simulation. For each panel, the upper part is the time series of the parallel electric field averaged in the (negative) gradient region; on the bottom part, the wavelet power spectrum is plotted as a function of the position along the magnetic field and the scales of field structures. The colour code, in logarithmic scale, is common to all panels. 
field power (see the spectrum at $\omega_{p e} t=760$ ). The last three panels reveal that these structures propagate toward the positive $z$ and quickly evolve. They also become dispersed in length scales: from an initial scale of $\sim 8 c / \omega_{p e}$, they go down to $\sim 5 c / \omega_{p e}$ and up to $\sim 30 c / \omega_{p e}$. This decay is the consequence of a complex evolution of the nonlinear structures which interacts with one another by crossing and merging. This particular aspect needs to be further investigated. This wavelet analysis is not able to discriminate between the different natures of the structures: weak double layer or bipolar, as both of them do have similar length scale and form in the same region.

\section{Discussion of the general wave/particle energy ex- change}

In Fig. 11 we present the parallel kinetic electron and wave energies as functions of time. On both curves, three different regions may be distinguished: a region of variations, a region of plateaus, and another region of variations. The first region corresponds to the formation of the parallel electric field and its installation over large spatial scales. This field accelerates electrons. After $\sim 100 \omega_{p e}^{-1}$ the wave to electron exchange rate is almost constant $(|\partial W / \partial t| \simeq$ cst in Fig. 11). This situation continues up to $\sim 500 \omega_{p e}^{-1}$, when the beam-plasma instability occurs and structures the field on small-scales. The second phase then begins when both energies plateau, which indicates that the wave/particle system reaches an equilibrium. This is the direct consequence of the action of the electron holes: they stop the acceleration process as they trap the particles in their electric field. This is illustrated by the vortex structures to which they are associated with in the electron phase space (see Fig. 9 and the analytic development in the Appendix). The weak double layers are known to be able to accelerate particles, as the net difference between their sides is not null. However, in our simulations, given their relative weakness and their small lifetime, a few tens of $\omega_{p e}^{-1}$, they would only be able to lead to electron acceleration if they were numerous enough along a given field line, which is not the case. Finally, as the electron holes dominate the field structure, the net result is a stop in the acceleration process. The associated plateaus in energies persist as long as these electron holes are sufficiently structured. When they decay (from $t=\sim 1000 \omega_{p e}^{-1}$ ), the large-scale electric field slowly overtakes the electron holes amplitude and then dominates the general field structure. Consequently, electron acceleration starts again, pumping wave energy. This second acceleration phase is, however, less efficient (the slope of the curve is smaller than in the first phase, before $t=500 \omega_{p e}^{-1}$ ), because the parallel electric field amplitude is smaller as wave dissipation occurred.

The Buneman instability has no noticeable influence on the wave and particle energies, although it presents some structures similar to the electron holes. In fact, it appears just before the beam-plasma instability, which is linked to the quenching of the wave/particle energy exchange, and it

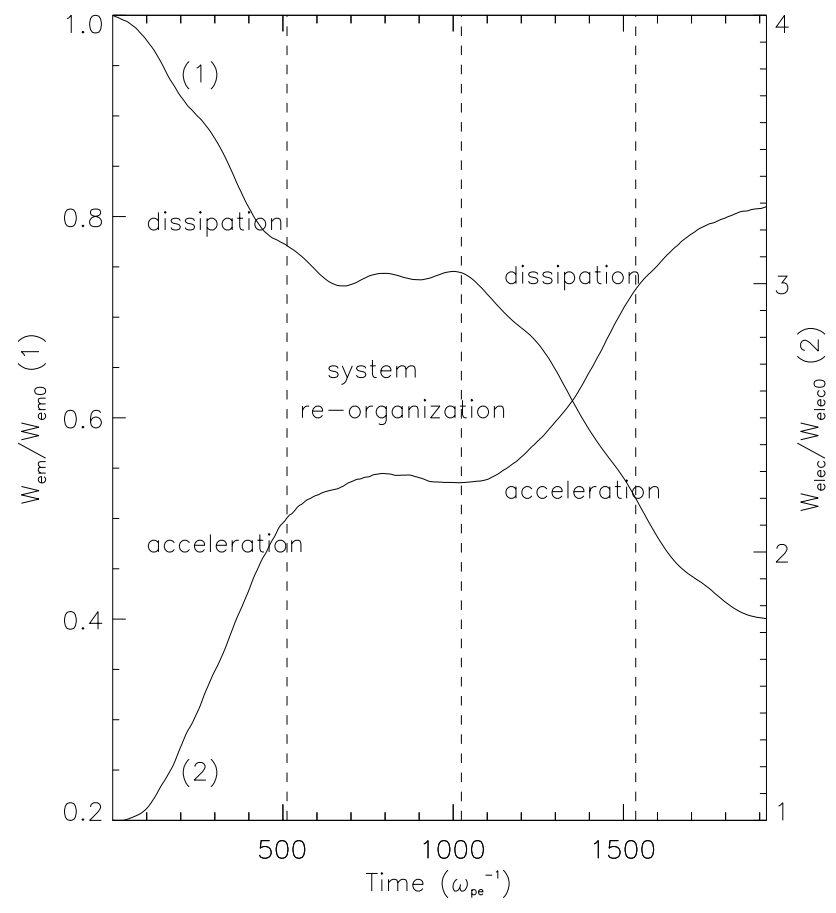

Fig. 11. Wave (label 1) and electron (label 2) energies as functions of time over $\sim 4$ Alfvén periods.

may be difficult to disentangle between the actions of both nonlinear instability-associated structures. Also, the associated wave perturbations do not overtake the large-scale field in amplitude (see $t=320 \omega_{p e}^{-1}$ in Fig. 10) as do the perturbations of electron holes.

In order to understand the energy history in the linear phase of the simulation, we performed comparisons with a periodic 1-D particle test model. We used a propagating sinusoidal electric field along the magnetic field direction, modeling the parallel electric field characteristics derived from the large amplitude simulation case (same velocity, same average amplitude). Electrons becomes accelerated by this field and their kinetic energy is recorded as a function of time. The energy curves are similar to PIC simulation ones for about one Alfvén period. Later, the nonlinearity associated with the beam-plasma instability, and the subsequent structuring of the field, prevent comparing both evolutions. Before this time, however, this simple linear model (electrostatic field, i.e. no potential vector, no magnetic gradient effects) is able to reproduce the characteristics of the electron energy. This confirms that the early stage of the simulation may be simply described in terms of an electrostatic moving accelerator.

Finally, we noticed that for $\delta B / B=0.025$ the wave energy decreases for one Alfvén period and then slightly increases for another period (evolution not shown). There is a corresponding feature on the electron energy which decreases on the second Alfvén period. In fact, high amplitude leads to electron trapping in the electrostatic structures, whereas lower amplitude does not lead to as much trapping because the sinusoidal part of the wave is still present: for lower amplitude the electrostatic structures do 
not completely overtake the linear long wavelength parallel electric field. When the linear field dominates, some particles are decelerated when they enter the positive part of $E_{\|}$ (ahead on the magnetic field line) which decreases the electron energy; as the total energy is conserved, the wave gains back energy. A similar behaviour is naturally observed with the 1-D model where no nonlinear effect intervenes. In conclusion, the nonlinearity plays an important role in the simulation as it blocks the return of energy from the particles towards the wave, a situation observed in either the 1-D model or the self-consistent simulation initialized with a small wave amplitude.

\section{Conclusions}

In this paper, we present results from simulations of a model which considers the interaction between Alfvén waves and transversely inhomogeneous plasma. Starting from initial conditions which mimic observational ones, numerous features observed in the auroral context have been selfconsistently reproduced: the presence of high energy electrons, the presence of electric field parallel to the ambient magnetic field, a high level of structure for both fields and phase space. In order to go beyond this observational aspect, several tools have been used to decompose the different processes at work. Thus, we emphasized the role of nonlinearities and instabilities in the rapid conversion from an initial low-frequency electromagnetic regime, to a highfrequency, electrostatic one. Therefore, this model offers a general framework in which electron acceleration and wave dissipation in inhomogeneous media may be explained.

We summarize below the main findings of the paper:

- The linear growth of the parallel electric field is found to provide an electron acceleration consistent with the one of a moving potential difference.

- The Buneman and weak beam-plasma instabilities have been identified by the program WHAMP. This analysis also rules out the possibility for these instabilities to be numerical.

- The amplitude of the incident Alfvén wave controls the system evolution; in the low amplitude case the Buneman instability is not triggered, whereas the beamplasma instability and the associated small-scale structures appear for high and low amplitudes. They appear more quickly in the high amplitude as the linear parallel electric field reaches larger values, and therefore leads earlier to the formation of an energetic electron beam.

- The evolution of wave and particle energies are shown to be exactly anti-correlated, emphasizing the energy exchange involved in the wave dissipation and electron acceleration processes. The energy deposition rate is constant in the linear acceleration phase, whereas it cancels out when particle trapping by nonlinear structures occurs.
- Two kinds of nonlinear structures are identified: weak double layers, observed in different plasma contexts, but here similar to the ones of the auroral regions; electron holes responsible for particle trapping.

Although the actual model possesses some limitations (periodical system, cautions when comparing simulation parameters and results with observed ones), it has the advantage to present, in a self-consistent way, a complex chain of processes which are usually exposed separately. In this extent, the ability to show the energy and regime conversions is a key point, whereas models tend generally to work in one regime or the other. Our model has been tested in the auroral context but it awaits application in other astrophysical contexts. Inhomogeneities and low frequency waves are indeed encountered in many environments. For instance, the model may be relevant in the ionosphere and in the tail of magnetospheres. Broadening the scope of the applications, the laboratory experimenters could make good use of such a model to explain their observations; on larger scales, we could also think of the external (non collisional) solar corona and galactic jets where mechanisms accounting for acceleration are needed. For the latter, there are indeed suggestions that an instability occurring at the base of the jets, where the density is inhomogeneous, is able to launch Alfvén waves (Varnière and Tagger, 2002); the subsequent interaction of these waves with the medium density may provide acceleration following processes analogous to those presented in our model.

\section{Appendix A Analytical model for the electron hole}

The electron phase space displayed in Fig. 8 exhibits a typical electron hole signature around $z \simeq 80$. We may reasonably expect the size of the phase space ring (the electron trap) to be related to the amplitude of the parallel electric. To demonstrate this we develop a simple electron hole model, in order to find an analytical expression which relates the amplitude of the trapping electric field to the size of the hole in the $\left(z, v_{z}\right)$ plane. This may give an indication of the physical process at work behind the complex behaviour exhibited on the left side of Fig. 9.

In the moving structure frame, we choose a sinusoidal electric field: $E_{\|}=-E_{0} \sin k z(t)\left(E_{0}>0\right)$, which will accelerate electrons located at $0<z<\pi / k$, and solve the reduced electron equation of motion:

$m \frac{\partial^{2} z}{\partial t^{2}}=e E_{0} \sin k z(t)$

with no asymptotic development of the sinus. It is a nonlinear equation similar to the one of the pendulum oscillation without the small angle approximation. Integrating for the velocity $v_{z}$ and position $z$ we obtain

$v_{z}=\frac{\partial z}{\partial t}=\sqrt{2(C-A \cos k z(t))}$ 
$r F(\delta, r)=k \sqrt{A} t$

with $A=\frac{e E_{0}}{m k}, r=\sqrt{\frac{2 A}{C+A}}, \quad \delta=\arcsin \left[\frac{(C+A)(1-\cos k z(t))}{2(C-A \cos k z(t))}\right]^{1 / 2}$, and $F$ is the elliptic integral of the first kind and $C$ is a constant which depends on the initial velocity. In Eq. (A3) the time $t$ is an explicit function of the position $z$.

In the case of a closed trajectory, we obtain from Eq. (A2):

$\Delta v_{z}=4 \sqrt{\frac{e E_{0}}{m k}}=4 \sqrt{\frac{e E_{0} \Delta z}{2 \pi m}}$

where $\Delta v_{z}$ is the width of the electron hole along the velocity axis. We expressed the extension along the $z$ axis by $\Delta z=\lambda=2 \pi / k$; Eq. (A4) relates the characteristic scales of the electron hole to one another. Following these equations, a plot of an electron trajectory in phase space is given on the right-hand side of Fig. 9.

We apply Eq. (A4) to the electron hole displayed in Fig. 8 (and enlarged in the left part of Fig. 9) and which travels at $v \simeq 0.6 c$. We measure: $\Delta z=7 c / \omega_{p e}$ and $\Delta v_{z}=0.5 c$ which leads to a normalized electric field of 0.014 ; this is consistent with maxima of the parallel electric field observed in the simulations when electron holes are developed (see also the fifth panel of Fig. 10). This simple analytic model is thus able to relate the characteristic scales of the electron hole to its sustaining electric field, coupling particle and field representations of the electron hole.

Acknowledgements. VG is supported by a UK PPARC grant. The numerical computations have been supported by IDRIS (CNRS computation center, France).

Topical Editor T. Pulkkinen thanks M. Volwerk and P. Kintner for their help in evaluating this paper.

\section{References}

Alfvén, H. and Fälthammar, C. G.: Cosmical Electrodynamics, 163, Clarendon, Oxford, 1963.

Andersson, L., Ergun, R. E., Newman, D. L., McFadden, J. P., Carlson, C. W., and Su, Y.-J.: Characteristics of parallel electric fields in the downward current region of the aurora, Physics of Plasmas, 9 (8), 109-130, 2002a.

Andersson, L., Wahlund, J.-E., Clemmons, J., Gustavsson, B., and Eliasson, L.: Electromagnetic waves and bursty electron acceleration: implications from Freja, Ann. Geophys., 20, 139, 2002b.

Bale, S. D., Kellogg, P. J., Larson, D. E., Lin, R. P., Goetz, K., and Lepping, R. P.: Bipolar electrostatic structures in the shock transition region: evidence of electron phase space holes, Geophys. Res. Lett., 25, 2929, 1998.

Bale, S. D., Hull, A., Larson, D. E., Lin, R. P., Muschietti, L., Kel$\log$, P. J., Goetz, K., and Monson, S. J.: Electrostatic turbulence and Debye-Scale structures associated with electron thermalization at collisionless shocks, Astrophys. J., 575, (1): L25-L28, Part 2, 2002.

Baronia, A. and Tiwari, M. S.: Kinetic Alfvén waves in an inhomogeneous anisotropic magnetoplasma in the presence of an inhomogeneous electric field: Particle aspect analysis, J. Plasma Physics, 63, 311, 2000.
Bingham, R., Bryant, D. A., and Hall, D. S.: A wave model for the aurora, Geophys. Res. Lett., 11, 327, 1984.

Bingham, R., Bryant, D. A., and Hall, D. S.: Auroral electron acceleration by lower-hybrid waves, Ann. Geophys., 6, 159, 1988.

Boström, R., Gustafsson, G., Holback, B., Holmgreen, G., Koskinen, H., and Kintner, P.: Characteristics of solitary waves and weak double layers in the magnetospheric plasma, Phys. Rev. Lett., 61, 82, 1988.

Bryant, D. A., Cook, A. C., Wang, Z.-S., de Angelis, U., and Perry, C. H.: Turbulent acceleration of auroral electrons, J. Geophys. Res., 96, 13 829, 1991.

Chaston, C. C., Bonnell, J. W., Peticolas, L. M., Carlson, C. W., McFadden, J. P., and Ergun, R. E.: Driven Alfvén waves and electron acceleration: A FAST case study, Geophys. Res. Lett., 29 (11), doi: 10.1029/2001GL013842, 2002.

Chen, L.-J. and Parks, G. K.: BGK electron solitary waves in 3D magnetized plasma, Geophys. Res. Lett., 29, (9) doi: 10.1029/2001GL013385, 2002.

Chiu, Y. T. and Schulz, M.: Self-consistent particle and parallel electrostatic field distributions in the magnetosphericionospheric auroral region, J. Geophys. Res., 83, 629, 1978.

Clarke, J. T., Ajello, J., Ballester, G., Ben Jaffel, L., Connerney, J., Gèrard, J.-C., Gladstone, G. R., Grodent, D., Pryor, W., Trauger, J., and Waite, J. H.: Ultraviolet emissions from the magnetic footprints of Io, Ganymede and Europa on Jupiter Nature, 415, 997, 2002.

Ergun, R. E., Su, Y.-J., Andersson, L., Carlson, C. W., McFadden, J. P., Mozer, F. S., Newman, D. L., Goldman, M. V., and Strangeway, R. J.: Direct observation of localized parallel electric fields in a space plasma, Phys. Rev. Lett., 87, 4, 2001.

Ergun, R. E., Andersson, L., Main, D. S., Su, Y.-J., Carlson, C. W., McFadden, J. P., and Mozer, F. S.: Parallel electric fields in the upward current region of the aurora: Indirect and direct observations, Phys. Plasma, 9, 3685, 2002a.

Ergun, R. E., Andersson, L., Main, D. S., Su, Y.-J., Newman, D. L., Goldman, M. V., Carlson, C. W., McFadden, J. P., and Mozer, F. S.: Parallel electric fields in the upward current region of the aurora: Numerical solutions, Phys. Plasma, 9, 3695, 2002b.

Franz, J. R., Kintner, P. M., Seyler, C. E., Pickett, J. S., and Scudder, J. D.: On the perpendicular scale of electron phase-space holes, Geophys. Res. Lett., 27, 169, 2000.

Gekelman, W., VanZeeland, M., Vincena, S., Palmer, N., Mitchell, C., and Dimonte, G.: Experiments on the propagation of Alfvén waves in plasmas with inhomogeneities and on Alfvén waves, which cause inhomogeneities, Proceedings of ISSS-6, 1-3, 2001.

Génot, V., Louarn, P., and Le Quéau, D.: A study of the propagation of Alfvén waves in the auroral density cavities, J. Geophys. Res., 104, 22 649, 1999.

Génot, V., Mottez, F., and Louarn, P.: Electron acceleration by Alfvén waves in density cavities, J. Geophys. Res., 105, 27 611, 2000.

Génot, V., Louarn, P., and Mottez, F.: Fast evolving spatial structure of auroral parallel electric fields, J. Geophys. Res., 106, 29633 , 2001.

Heyvaerts, J. and Priest, E. R.: Coronal heating by phase-mixed shear Alfvén waves, Astron. Astrophys., 117, 220, 1983.

Janhunen, P. and Olsson, A.: New model for auroral acceleration: O-shaped potential structure cooperating with waves, Ann. Geophys., 18, 596, 2000.

Keiling, A., Wygant, J. R., Cattell, C., Peria, W., Parks, G., Temerin, M., Mozer, F. S., Russell, C. T., and Kletzing, C. A.: Correlation of Alfvén wave Poynting flux in the plasma sheet at $4-7 R_{E}$ with 
ionospheric electron energy flux, J. Geophys. Res., 107 (A7) , doi: 10.1029/2001JA900140, 2002.

Keiling, A., Wygant, J. R., Cattell, C. A., Mozer, F. S., and Russell, C. T.: The global morphology of wave Poynting flux: Powering the aurora, Nature, 299, 383, 2003.

Le Contel, O., Pellat, R., and Roux, A.: Self-consistent quasi-static parallel electric field associated with substorm growth phase, J. Geophys. Res., 105, 12 945, 2000.

Louarn, P., Wahlund, J. E., Chust, T., de Feraudy, H., Roux, A., Holback, B., Dovner, P. O., Eriksson, A. I., and Holmgren, G.: Observation of kinetic Alfvén waves by the Freja spacecraft, Geophys. Res. Lett., 21, 17, 1994.

Lysak, R. L. and Song, Y.: Kinetic theory of the Alfvén wave acceleration of auroral electrons, J. Geophys. Res., 108, (A4), 8005, doi: 10.1029/2002JA009406, 2003.

Mälkki, A., Eriksson, A. I., Dovner, P. O., Boström, R., Holback, B., Holmgren, G., and Koskinen, H. E. J.: A statistical survey of auroral solitary waves and weak double layers, Occurrence and net voltage, J. Geophys. Res., 98, 15 521, 1993.

Mandrake, L., Pritchett, P. L., and Coroniti, F. V.: Electron beam generated solitary structures in a nonuniform plasma system, Geophys. Res. Lett., 27, 2869, 2000.

Mangeney, A., Salem, C., Lacombe, C., Bougeret, J.-L., Perche, C., Manning, R., Kellogg, P. J., Goetz, K., Monson, S. J., and Bosqued, J.-M.: WIND observations of coherent electrostatic waves in the solar wind, Ann. Geophys., 17, 307, 1999.

Marklund, G. T., Ivchenko, N., Karlsson, T., Fazakerley, A., Dunlop, M., Lindqvist, P.-A., Buchert, S., Owen, C., Taylor, M., Vaivads, A., Carter, P., André, M., and Balogh, A.: Temporal evolution of the electric field accelerating electrons away from the auroral ionosphere, Nature, 414, 724, 2001.

Miura, A. and Sato, T.: Numerical simulation of global formation of auroral arcs, J. Geophys. Res., 85, 73, 1980.

Miyake, T., Omura, Y., and Matsumoto, H.: Electrostatic particle simulations of solitary waves in the auroral region, J. Geophys. Res., 105, 23 239, 2000.

Mottez, F., Chanteur, G., and Roux, A.: Filamentation of plasma in the auroral region by an ion-ion instability: A process for the formation of bidimensional potential structures, J. Geophys. Res., 97, $10801,1998$.

Mottez, F., Adam, J. C., and Heron, A.: A new guiding centre PIC scheme for electromagnetic highly magnetized plasma simulation, Computer Physics Communications, 113 (2-3): 109-130, 1998.

Newman, D. L., Goldman, M. V., Ergun, R. E., and Mangeney, A.: Formation of double layers and electron holes in a current-driven space plasma, Phys. Rev. Lett., 87, 25, 2001.
Noël, J.-M., St.-Maurice, J.-P., and Blelly, P.-L.: Nonlinear model of short-scale electrodynamics in the auroral ionosphere, Ann. Geophys., 18, 1128, 2000.

Omura, Y., Matsumoto, H., Miyake, T., and Kojima, H.: Electron beam instabilities as generation mechanism of electrostatic solitary waves in the magnetotail, J. Geophys. Res., 101, 2685, 1996.

Omura, Y., Kojima, H., Umeda, T., and Matsumoto, H.: Observational evidence of dissipative small-scale processes: Geotail spacecraft observations and simulation of electrostatic solitary waves, Astrophys. Space Sc., 277, 45, 2001.

Papadopoulos, K.: A review of anomalous resistivity for the ionosphere, Rev. Geophys. Spac. Res., 15, 113, 1977.

Rönnmark, K.: WHAMP: Waves in Homogeneous, Anisotropic Multicomponent Plasmas, Rep. 179, Kiruna Geophys. Inst., Kiruna, Sweden, 1982.

Ruderman, M. S., Goldstein, M. L., Roberts, D. A., Deane, A., and Ofman, L.: Alfvén wave phase mixing driven by velocity shear in two-dimensional open magnetic configuration, J. Geophys. Res., 104, 17 057, 1999.

Seyler, C. E., Wahlund, J. E., and Holback, B.: Theory and simulation of low-frequency plasma waves and comparison to Freja satellite observations, J. Geophys. Res., 100, 21 453, 1995.

Silberstein, M. and Otani, N. F.: Computer simulation of Alfvén waves and double layers along auroral magnetic field lines, J. Geophys. Res., 99, 6351, 1994.

Singh, N., Loo, S. M., Wells, B. E., and Deverapalli, C.: Threedimensional structure of electron holes driven by an electron beam, Geophys. Res. Lett., 27, 2469, 2000.

Temerin, M., Cerny, K., Lotko, W., and Mozer, F. S.: Observations of double layers and solitary waves in auroral plasma, Phys. Rev. Lett., 48, 1175, 1982.

Torrence, C. and Compo, G. P.: A practical guide to wavelet analysis, Bull. Amer. Meteor. Soc., 79, 61, 1998.

Umeda, T., Omura, Y., Matsumoto, H., and Usui, H.: Formation of electrostatic solitary waves in space plasmas: Particle simulations with open boundary conditions, J. Geophys. Res., 107 (A12), 1449, doi:10.1029/2001JA000286, 2002.

Varnière, P. and Tagger, M.: Accretion-ejection instability in magnetized disks: Feeding the corona with Alfvén waves, Astron. Astrophys., 394, 329, 2002.

Volwerk, M., Louarn, P., Chust, T., Roux, A., and de Féraudy, H.: Solitary kinetic Alfvén waves: A study of the Poynting flux, J. Geophys. Res., 101, 13 335, 1996. 